\title{
Citespace-based Analysis of Recent Status of Information Systems Social Sicence Papers of China and Others
}

\author{
Haoyuan Lan*, Hui Du
}

Beijing Jiaotong University

*Corresponding author. Email: 17711011@bjtu.edu.cn

\begin{abstract}
To comprehensively and deeply understand recent status of information systems social science papers of China and others, following the general process of mapping knowledge domains analysis, firstly, papers on information systems from CSSCI and SSCI in the last five years were searched and cleaned respectively. Secondly, with the help of the analysis software Citespace, mapping knowledge domains were drawn, interpreted and compared. In addition to recent research status of China and other countries, especially useful reference for Chinese research was provided.
\end{abstract}

Keywords: Mapping Knowledge Domains, Information Systems, Citespace, Social Science, Recent Status Analysis.

\section{基于Citespace的中外信息系统社会科学论文近况分析 蓝浩源, 杜晖}

北京交通大学, 北京, 中国

*通讯作者. 由阝箱: 17711011@bjtu.edu.cn

中文摘要

为全面深入了解中外信息系统领域社会科学论文近况, 遵循科学知识图谱分析的一般流程, 检索和清洗了来自 CSSCI和SSCI中近五年的信息系统论文。借助分析软件Citespace绘制了图谱并进行了解读和中外比较。提供中 外研究近况的同时, 特别为中国研究提供了有益参考。

关键词: 科学知识图谱；信息系统：Citespace；社会科学；近况分析.

\section{1. 引言}

科学知识图谱可以揭示学科发展状况、研究热点 以及前沿领域等[1], 其一般分析流程也已趋于共识 [2]。Citespace是当前应用最为广泛的科学知识图谱分 析软件, 被广泛应用在不同学科的科学知识图谱分析 中[3]。其中, 苏玥竹[4]利用从WoS (Web of Science) 搜集的2003至2012年美国的信息系统文献，通过关键 词、研究机构、作者和文献的中心度分析, 发现对未
来研究具有指导作用的十篇最主要文献以及美国的 研究热点。李娜娜[5]通过分析从CNKI (China National Knowledge Infrastructure) 提取的18种GIS (Geographic Information System）期刊论文图谱，发现作者在2011 至2015年更趋向合作并在一些热点研究方面趋近成 熟。Zong Fengzou等[6] 借助从SCI和SSCI收集的876 篇商业智能文献的关键词聚类分析，发现1997至2017 年的研究热点是云计算。但是，上述研究所用文献不 
能反映近况且没有集中在信息系统领域的社会科学 论文，也没有进行中外比较。

本文结构如下: 第2节介绍了数据的准备情况。第 3节分别从作者-机构共现，关键词聚类，以及关键词 时区方面进行了中外近 5 年数据的分析。最后, 在第 4 节总结了全文并指出了存在的不足。

\section{2. 数据准备}

CSSCI（Chinese Social Sciences Citation Index）论 文从CNKI以Refwork格式导出, 检索条件为“篇名”或 “关键词”或“摘要”包含“管理信息系统”，发文时间为 2016至2021年, 文献类型为“期刊论文”。SSCI (Social Sciences Citation Index）论文从WoS中以纯文本格式 导出, 包含所有记录来源, 选取“核心数据库合集”, 检索条件为“篇名”或 “关键词”或 “摘要”包含 “Management Information System” 或 “Management Information Systems”, 发文时间也为2016至2021年, 文献类型为“Article”。经过清洗（去重以及去除征稿 启事) 得到CSSCI论文95篇, SSCI论文207篇。

将清洗后的数据以download_x.txt形式重命名并创 建项目文件夹后, 导入Citespace进行标准化（CSSCI 论文选择 CNKI Format, SSCI 论文选择 Remove Duplicates (WoS)), 然后选择关联强度最强的Cosine 系数和 TopN, 选择 Pathfinder 和 Pruning sliced networks[7]相结合的简化方式。

\section{3. 数据分析}

\section{1. 作者-机构共现分析}

1. 根据CSSCI论文得到的共现网络如图1所示, 可 以看出: 存在发文量较多的机构, 前三名分别是中国 科学技术信息研究所、南京大学信息管理学院和武汉 大学信息资源研究中心; 也存在发文量较多的作者,
前三名分别是袁勤俭、马友华、王静。虽然中国科学 技术信息研究所和武汉大学信息资源研究中心拥有 多位作者, 但却没有发文量较多的作者; 南京大学信 息管理学院拥有发文量最多的作者袁勤俭, 且与朱哲 慧和颜祥林有合作关系; 作者马友华隶属于多个机构, 并且与隶属不同机构的王静等作者存在合作关系。只 有少数作者和机构间存在合作关系。大部分作者都独 立或只隶属于一个机构。

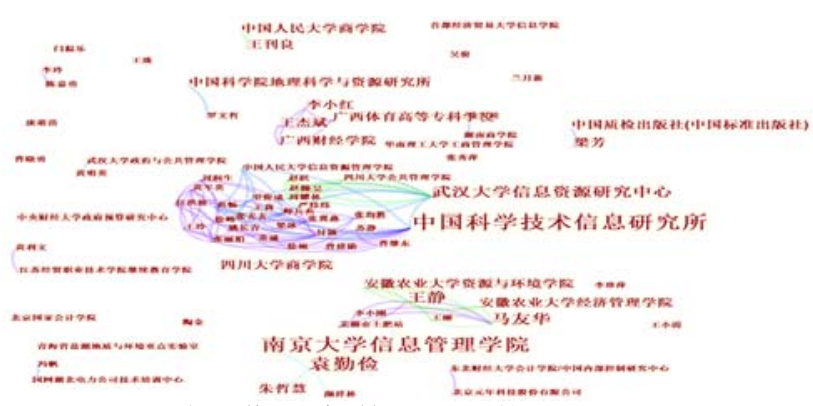

图1 CSSCI论文作者-机构共现网络

2. 根据SSCI论文得到的共现网络如图2所示, 可以 看出: 存在发文量较多的机构, 前三名分别是Minist Hlth、London Sch Hyg \& Trop Med和Ctr Dis Control \& Prevent; 也存在发文量较多的作者, 前两名分别是 Molly Brown和Manuela De Allegri。图中一端连接 Minist Hlth的红色线段另一端是隶属于该机构的作者, 一端连接London Sch Hyg \& Trop Med的紫色线段另 一端是隶属于该机构的作者, 一端连接 Ctr Dis Control \& Prevent的黄色线段另一端是隶属于该机构 的作者。以上三家机构的发文作者非常多，但却没有 发文量较多的作者。发文量较多的作者Molly Brown 隶属于多个机构, 也与其他多个作者存在合作关系, 而Manuela De Allegri则与更多的作者存在合作关系。 大部分机构和作者间都存在合作关系。多数作者隶属 于一个机构, 少数作者隶属于多个机构。

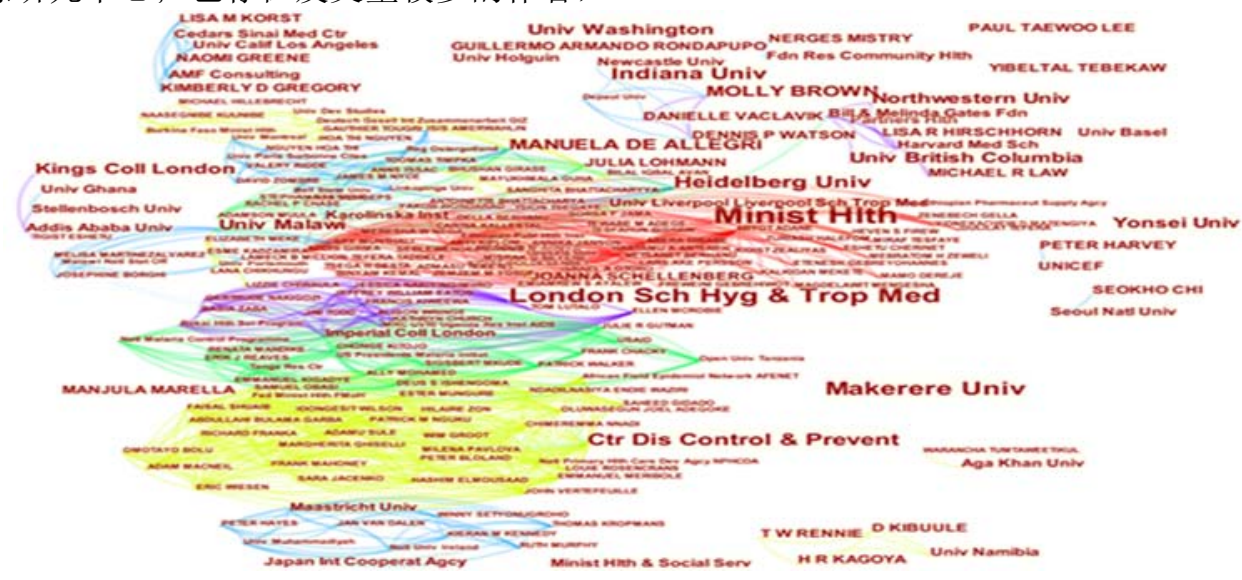

图2 SSCI论文作者-机构共现网络

3. 该将图1和图2进行比较发现: 中外都存在发文 量较多的机构和作者, 存在机构间以及同一机构内和 不同机构间的作者合作，一位作者可以隶属于多个机
构。但是，更多国外机构拥有的发文作者多，作者间 的合作比国内多。中国大部分机构和作者相对独立, 存在发文量多的机构拥有发文量多的作者而国外没 有。 


\section{2. 关键词聚类分析}

1. 根据CSSCI论文得到的关键词聚类网络如图3所 示, 可以看出: 按关键词数量从多到少的 4 个聚类分 别是管理信息系统、科技情报工作、空间量化和新经 济，代表中国管理信息系统研究的4个主要方向。

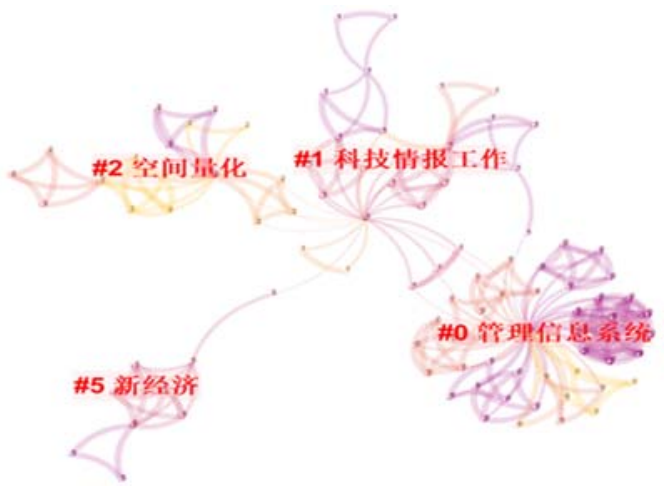

图3 CSSCI论文关键词聚类网络

2. 根据SSCI论文得到的关键词聚类网络如图4所 示, 可以看出: 按关键词数量从多到少的顺序排列共 有 18 个聚类, 代表了国外管理信息系统研究的 18 个主 要方向。

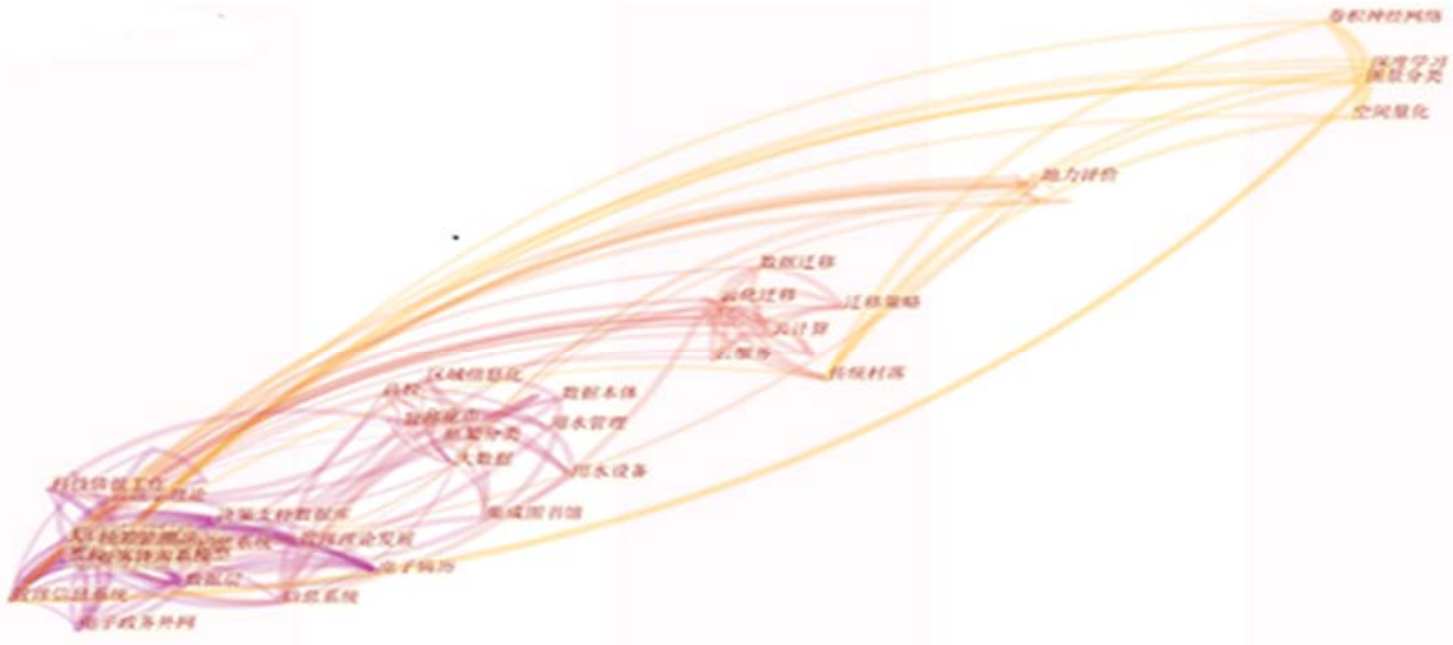

图5 CSSCI论文关键词时区网络

2. 根据SSCI论文得到的关键词时区网络如图6所 示, 可以看出: 关键词数量有减少趋势。在2016至2019 年致力于向不同主题扩展, 之后更倾向于已有主题的 深入。2016至2017年的关键词包括 technology、 homelessness、disease、education policy、risk factor、 computer game、forecasting、health economy 等, 2018

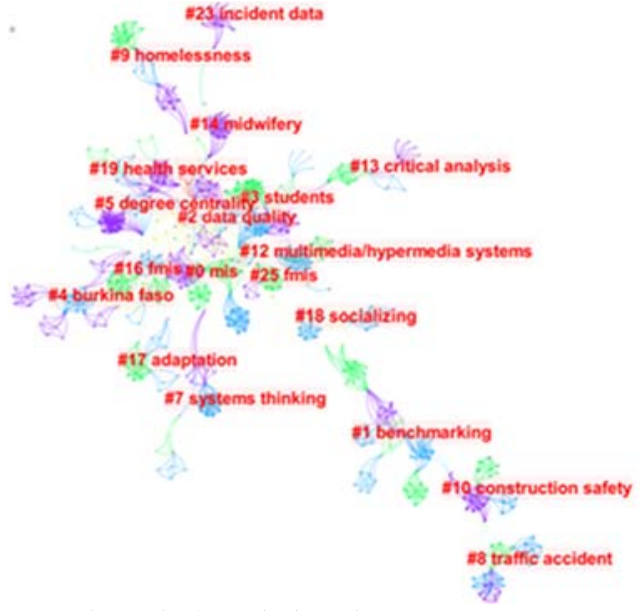

图4 SSCI论文关键词聚类网络

3. 将图3和图4进行比较发现: 中外都存在相同聚 类“管理信息系统”。但是, 中国的聚类数量远不及外 国，除了“管理信息系统“外，不存在相同的聚类。既 说中外研究的主题不同, 也说明国外研究的主题更加 丰富。

\section{3. 关键词时区图分析}

1. 根据CSSCI论文得到的关键词时区网络如图5所 示, 可以看出: 关键词数量有减少趋势。2016至2017 年向不同主题扩展，之后更倾向于已有主题的深入。
至2019年的关键词包括 mental health problem、supply chain management、 job market skill、farm performance、 bullying behavior等, 2020迄今的关键词包括 health management information system、 information sharing、 tanzania、routine health information system 等。 


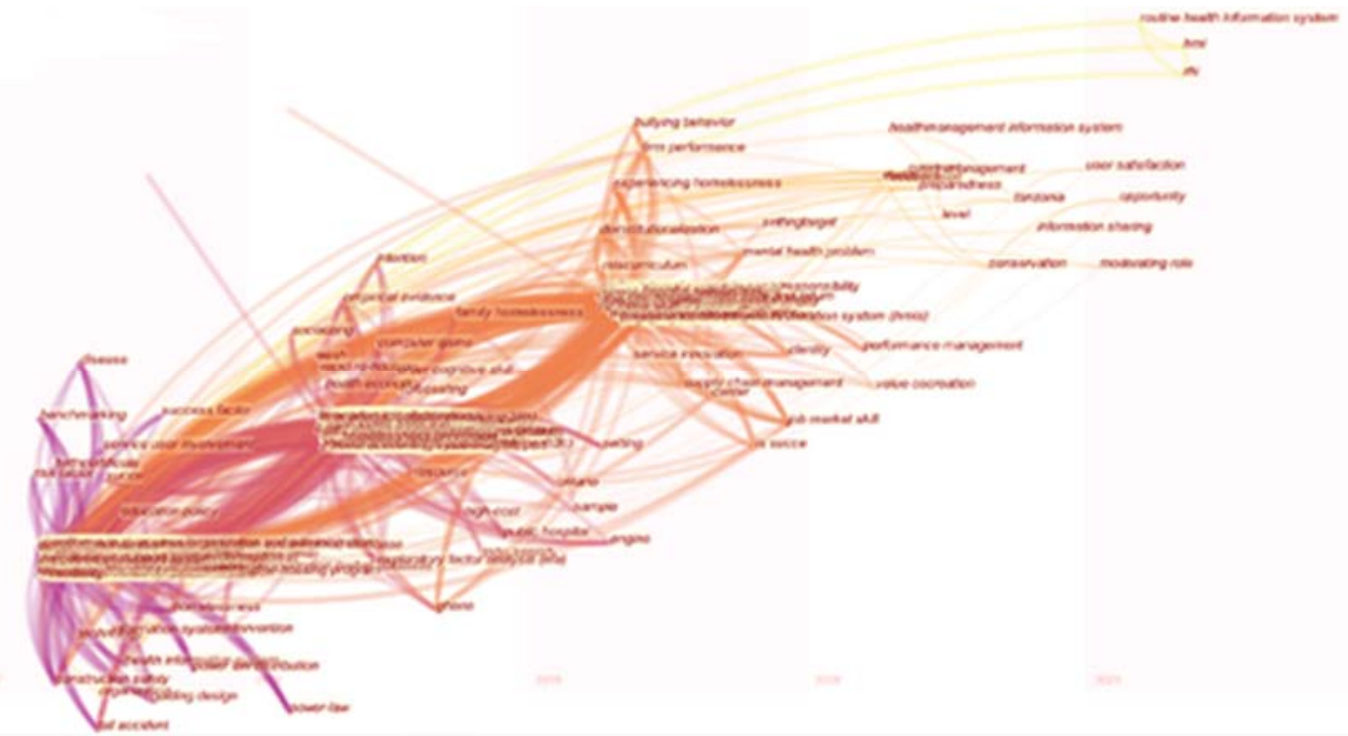

图6 SSCI论文关键词时区网络

3. 将图5和图6进行对比发现: 两者的关键词数量 都有逐年减少趋势, 都从向对不同主题的扩展到对已 有主题的深入过程。但是，除了2020年迄今，外国的 关键词数量明显多于中国, 说明国外研究的主题远超 中国。

\section{4. 结论}

遵循科学知识图谱分析的一般流程, 检索和清洗 了来自CSSCI和SSCI中近五年的管理信息系统论文。 借助分析软件Citespace绘制了图谱并进行了解读和 中外比较。提供中外研究近况的同时, 特别为未来的 中国研究提供了有益参考。

由于管理信息系统涉及主题众多, 文献形式多样, 因此, 本文用于分析的数据并不全面。另外, 由于专 业知识和能力的限制, 对图谱的解读和对比也较肤浅。

\section{REFERENCES}

[1] Jie Jin, Tingting Li and Zhongxing Wang (2020), Cognitive science analysis based on Citespace knowledge visualization (eds.), The Seventeenth Proceedings of the Annual Meeting of Shenyang Science (pp.769-774). DOI: 10.26914/c.cnkihy.2020.012062.

[2] Siluo Yang and Ruizhen Han (2012), Analysis of Foreigh Methods and Tools of Mapping Knowledge Domain, Documentation, Information \& Knowledge (06), 101-109. DOI: 10.13366/j.dik.2012.06.006.

[3] ZeWen Hu, Jianjun Sun and Yishan Wu (2013), Research Review on Application of Knowledge Mapping in China, Library and Information Service (03), 131-137+84. DOI: CNKI: SUN: TSQB.0.2013-03-025.
[4] Yuezhu Su (2014), Research Status - quo Analysis of IS in the last ten Years of U.S. based on Mapping Knowledge, Journal of Modern Information (08), 154-159. DOI: CNKI: SUN: XDQB.0.2014-08-031.

[5] Nana Li (2018), Analysis on the Research Status of Geographic Information System in China in Recent Five Years: Bibliometric Analysis Based on Citespace, Geomatics \& Spatial Information Technology (07), 168-171+175+179. DOI: CNKI: SUN: DBCH.0.2018- 07-047.

[6] Zongfeng Zou, Jie Cheng, Kugan Huang and Yonghan Gui, Research on the Developments of Business Intelligence and its Enlightenment based on Bibliometric Statistics and Knowledge Map Analysis, Journal of Physics: Conference Series, Volume 1176, Issue 4, 2019.

[7] Chen, C. and Morris, S (2003), Visualizing evolving networks: Minimum spanning trees versus Pathfinder networks, Proceedings of IEEE Symposium on Information Visualization, (Seattle, Washington, 2003), IEEE Computer Society Press, 67-74. 